\title{
Development of A-Stable block method for the solution of stiff ordinary differential equations
}

\begin{abstract}
A fixed step-size multistep block method for stiff Ordinary Differential Equations (ODEs) using the 2-point Block Backward Differentiation Formulas (BBDF) with improved efficiency is established. The method is developed using Taylor's series expansion. The order and the error constant of the method are determined. To validate the new method is suitable for solving stiff ODEs, the stability and convergence properties are discussed. Numerical results indicate that the new method produced better accuracy than the existing methods when sloving the same problems.
\end{abstract}

Keyword: Block method; Backward differentiation formula; Stiff, Stability; Ordinary differential equations 